1 OpenAPS

2 The BMJ

Corresponding author:

Dana@OpenAPS.org

Cite this as: $B M J$ 2021;375:n2891

http://dx.doi.org/10.1136/bmj.n2891

Published: 24 November 2021

\title{
We need more patient and public reviews on research papers-and the resources to do so
}

Dana M Lewis, ${ }^{1}$ Emma Doble ${ }^{2}$

Historically, patients have always been considered the passive recipients of healthcare. This way of thinking affected everything from how people were cared for in a clinician's office or hospital bed, to how they participated in clinical trials. It's also meant that patients have previously had no role in the production or review of medical literature after research has been completed. However, this is changing, and now patients and members of the public are increasingly involved in new and meaningful ways at more steps in the research process, including as potential reviewers of medical papers. This has enormous benefits for science and healthcare. But patients and members of the public are not always provided with the relevant resources to participate effectively and efficiently, and this is something that journals need to work on.

\section{Better guidance could increase participation}

In 2014, The BMJ started soliciting patient and public (or P\&P) reviews of papers. ${ }^{1}$ The role of patients and public reviewers in health research cannot be underestimated, with patients and members of the public bringing a unique and valuable perspective to research and peer review. ${ }^{2}$ However, as with traditional novice peer reviewers, patients and public reviewers often have little guidance on the process of peer review and do not receive feedback on the reviews they give. This can leave reviewers feeling unable to contribute to peer review or unlikely to review again if the process has been unsatisfactory. ${ }^{3}$

Patient and public review involves people with lived experience peer reviewing papers alongside traditional peer review. Where guidance for peer reviewers is given, it is often limited, journal specific, and very often restricted to the process of submitting a peer review within the journal's electronic system, rather than guidance focusing on the content of the review. In August 2021 BMJ Health \& Care Informatics published a "A Step-By-Step Guide to Peer Review: A Template for Patients \& Novice Reviewers." ${ }^{4}$ This template gives an outline of the peer review process and answers key questions, which patients and members of the public can use and refer to when completing a peer review. It also gives some direction on what to expect during the peer review process. This guidance is helpful not only for patients and public reviewers, but also for traditional reviewers who are new to peer review.

One area that is still left as a "grey area" for patient and novice reviewers is how to provide the overall review rating for an article. Many journals will ask reviewers to provide a recommendation as to whether the paper should require major or minor revisions. This terminology can be new to patients and public reviewers and understanding what counts as major versus minor revisions may be unclear. Providing some context or examples of what would constitute a major or minor revision might help patients and public reviewers consider this aspect of their review. Minor revisions might include grammar/plain language suggestions, recommendations for additional citations, or small additions to the overall content. Major revisions are more likely to include significant additions of content, larger revisions to the flow or order of content within the article, or additional scientific analysis of included data.

It should be made clear to patients and public reviewers that their review is taken into consideration alongside those by other traditional peer reviews, for the editors to make the final decision about whether the article is accepted (with varying levels of revisions) or rejected. Their review alone is not the deciding factor.

We also think it's useful for patients and public reviewers to consider the language and tone of their review feedback, and to think about how they would feel reading or receiving the same review on an article they had submitted. (This is also advice we would give to traditional peer reviewers!)

\section{The work of patient and public reviewers should be funded}

Guidance is a positive start, but it isn't necessarily enough in and of itself to solve the challenges for all patient and public reviewers. Some journal publishers provide general tips for peer review, but patient reviewers may benefit from more hands-on, specific guides or tip sheets from the perspectives of patient reviewers.

Of course, guidance isn't the only resource needed. Patient and public review programmes should also be funded. The new Patient and Partner Initiative from BMJ Health \& Care Informatics recently introduced a model ${ }^{5}$ where reviewers receive funding for their time and expertise in recognition of their contribution and improvement of the medical literature. ${ }^{6}$ Unlike traditional reviewers, patient and public reviewers do not necessarily have day jobs where this work is seen as part of their "service" portfolio and an expected part of their role. Journals creating new patient and public partnership programmes or patient and public review programmes should also designate funding towards those schemes. This ensures opportunities for equitable participation and true diversity of experience from those with lived experience, rather than selecting for those with the privilege and circumstances which permit participation within a messy system. 


\section{A virtuous circle}

A lot of people stand to benefit from the changes we've suggested. Improving the process and guidance given to patient reviewers will also, in turn, improve the process for novice traditional reviewers. The better the experience that peer reviewers have (including funding for their time and expertise), the more likely they are to continue to contribute as reviewers when invited in the future. Creating this positive cycle will increase the quality of reviews and medical literature overall, thus ultimately improving healthcare for everyone.

Competing interests: Emma Doble is a patient editor at The BMJ and is a member of the BMJ Health \& Care Informatics patient and public involvement sub-committee. Dana Lewis is a volunteer member of the BMJ Health \& Care Informatics patient and public involvement sub-committee. Nothing further declared.

1 Patient and public partnership. The BMJ. https://www.bmj.com/campaign/patient-partnership

2 Boivin A, Richards T, Forsythe L, etal. Evaluating patient and public involvement in research. BMJ 2018;363:k5147. doi: 10.1136/bmj.k5147. pmid: 30522999

3 Schroter S, Price A, Flemyng E, etal. Perspectives on involvement in the peer-review process: surveys of patient and public reviewers at two journals. BMJ Open 2018;8:e023357. doi: 10.1136/bmjopen-2018-023357. pmid: 30185581

4 Salmi L, Blease C. A step-by-step guide to peer review: a template for patients and novice reviewers. BMJ Health Care Inform 2021;28:e100392. doi: 10.1136/bmjhci-2021-100392. pmid: 34413120

5 Lewis D, Scott P. BMJ HCI launches partnership programme for patients and carers as authors and peer reviewers. BMJ Health Care Inform 2021;28:e100471. doi: 10.1136/bmihci-2021-100471. pmid: 34799412

6 Patient and public involvement. BMJ Health \& Care Informatics. https://informatics.bmj.com/pages/patient-and-public-involvement/ 